\title{
Respiratory muscle senescence in ageing and chronic lung diseases
}

\author{
Joaquim Gea, Pilar Ausín, Juana Ma Martínez-Llorens (i) and Esther Barreiro \\ Affiliation: Dept of Respiratory Medicine, Hospital del Mar - IMIM, DCEXS, Universitat Pompeu Fabra,
} CIBERES, ISCIII, Barcelona Respiratory Network, Barcelona, Spain.

Correspondence: Joaquim Gea, Respiratory Medicine Dept, Hospital del Mar, Pg Marítim 27, Barcelona, 08003 Spain. E-mail: quim.geađupf.edu

@ERSpublications

Ageing leads to loss of respiratory muscle function, mostly through changes in respiratory mechanics, oxidative stress, probable mild muscle atrophy, excitation/contraction uncoupling and impaired bioenergetics. Premature senescence can also be present. https://bit.ly/32n4gkR

Cite this article as: Gea J, Ausín P, Martínez-Llorens JM, et al. Respiratory muscle senescence in ageing and chronic lung diseases. Eur Respir Rev 2020; 29: 200087 [https://doi.org/10.1183/16000617.0087-2020].

ABSTRACT Ageing is a progressive condition that usually leads to the loss of physiological properties. This process is also present in respiratory muscles, which are affected by both senescent changes occurring in the whole organism and those that are more specific for muscles. The mechanisms of the latter changes include oxidative stress, decrease in neurotrophic factors and DNA abnormalities. Ageing normally coexists with comorbidities, including respiratory diseases, which further deteriorate the structure and function of respiratory muscles. In this context, changes intrinsic to ageing become enhanced by more specific factors such as the impairment in lung mechanics and gas exchange, exacerbations and hypoxia. Hypoxia in particular has a direct effect on muscles, mainly through the expression of inducible factors (hypoxic-inducible factor), and can result in oxidative stress and changes in DNA, decrease in mitochondrial biogenesis and defects in the tissue repair mechanisms. Intense exercise can also cause damage in respiratory muscles of elderly respiratory patients, but this can be followed by tissue repair and remodelling. However, ageing interferes with muscle repair by tampering with the function of satellite cells, mainly due to oxidative stress, DNA damage and epigenetic mechanisms. In addition to the normal process of ageing, stress-induced premature senescence can also occur, involving changes in the expression of multiple genes but without modifications in telomere length.

\section{Ageing and muscles}

Ageing is a universal and progressive condition that usually leads to the loss of physiological properties in cells, tissues, organs, systems and individuals. The progressive ageing of the overall population in developed countries gives it a special importance, and also entails the association with numerous comorbidities. Among the various tissues that undergo changes with ageing is the skeletal muscle, which is characterised by its contractile properties and essentially by resulting in limb movements by bringing bone elements closer through the joints. However, there is a group of muscles whose contractile function produces a different effect, the generation of changes in intrathoracic pressures that allows the airflow: these are the respiratory muscles, which are also skeletal muscles. This means that their structure includes fascicles, and these in turn are composed of muscle fibres (which are multinucleate and post-mitotic cells), containing myofibrils and the contractile units (sarcomere) [1]. The main molecules that make up the sarcomere and participate in the contraction are myosin (sarcomere thick filament) and actin (sarcomere thin filament), although other proteins are also very important. Muscle fibres can be divided into type I

Provenance: Commissioned article, peer reviewed

Received: 6 April 2020 | Accepted after revision: 9 July 2020

Copyright $\odot$ ERS 2020. This article is open access and distributed under the terms of the Creative Commons Attribution Non-Commercial Licence 4.0 . 
and type II, the latter in turn being subdivided into IIa and IIx. This classification depends on their molecular components (mostly the predominant myosin heavy chain (MyHC) isoform) and metabolic and functional properties. Thus, type I fibres (with MyHC-I) have predominantly aerobic metabolism, show a relatively slow contraction and are highly resistant to fatigue. Type IIx (with MyHC IIx) in contrast, are fundamentally anaerobic, have a fast and powerful contraction, although they become easily fatigued. Characteristics of type IIa fibres are intermediate between those of type I and type IIx. Interestingly, type IIb fibres (and their corresponding MhHC-IIb), which have a very fast contraction, are present in rodents and other mammals but not in humans. Muscle fibres, being cells without the ability to divide, depend on a special group of stem cells called satellite cells for their maintenance and repair, which are usually inactive (quiescent) unless required [1].

\section{Muscle function}

From a functional point of view, skeletal muscles have two main characteristics, the strength or the mechanical expression of its maximum contractile capacity, and the resistance or ability to maintain a submaximal effort over time [2]. Strength depends fundamentally on muscle mass, while resistance is a function of the aerobic capacity of the muscle, which in turn requires the adequate participation of various elements: total blood volume and local blood flow; haemoglobin concentration; fibre type predominance; capillary and mitochondrial density; myoglobin; and aerobic enzyme content. Failure of one or both of these physiological characteristics is known as muscle dysfunction, where it is possible to differentiate between weakness and fatigue [2]. The former is a relatively permanent state that can only be reversed with medium or long-term measures, while the latter is a transitory dysfunction that can be easily solved with rest (figure 1). Although other definitions of fatigue exist, they are not very operative from a clinical point of view. The weakness and/or fatigue of limb muscles leads to difficulties in the exercise capacity and physical activity of patients, with important limitations in their daily life. However, respiratory muscle dysfunction is even more serious, since it not only leads to dyspnoea, impairment in the mechanics of the respiratory system, hypoxia and hypercapnia, with an important limitation in the physical activity of the patient, but can also lead to their death. If the involvement of these muscles is mild-to-moderate, their condition can be improved with nutritional supplements, specific training (such as programmes providing adequate loads through a threshold valve), or even anabolic drugs, although the latter have not shown clear results. Nevertheless, when respiratory muscle weakness or fatigue leads the patient to a critical situation, it is possible to obtain their rest through the various modalities of respiratory support $[1,2]$. This includes invasive and noninvasive mechanical ventilation as well, and most probably high flow nasal cannula.

\section{Ageing}

Ageing also occurs in muscle tissue, being determined by genetic factors (including general genetic background, sex and ethnicity), body development in the early stages of growth, lifestyle (toxic habits such as tobacco and alcohol, physical activity, diet, etc.) and the presence of comorbidities, among which respiratory diseases and associated circumstances (e.g. hypoxia, systemic inflammation, oxidative stress) $[3,4]$ can be found (figure 2). All these factors can condition a greater or lesser degree of structural and functional changes, leading to a reduction in the subject's physiological capacity, and potentially to disability, frailty, loss of independence and a marked impairment in the quality of life [3]. It is obvious that there is a physiological process of senescence, the so-called healthy ageing (figure 3). This is also characterised by muscle functional loss but within limits considered as normal. In this regard, it is known that, especially from the age of 50 years, subjects lose up to $2 \%$ of their muscle mass and up to $3 \%$ of their contraction force per year [5]. The loss of muscle mass is not always evident through changes in body weight, as it can be disguised by a parallel increase in fat mass (sarcopenic obesity) [5]. Hence the importance of determining the percentage of lean mass in clinics for detection of sarcopenia, and not limiting the control of the nutritional status only to the assessment of body weight [2]. It is accepted that 9-18\% of subjects are sarcopenic at the age of 65 years, reaching $30 \%$ from the age of 80 years [6]. Regarding the loss of strength, functional limitations appear when it attains a $30 \%$ decrease, and disability if it is $>70 \%$ [3]. Fortunately, these declines can be positively modulated with a good level of physical activity and/or training. In contrast, sedentary lifestyle or immobilisation (e.g. that due to bone fractures) determine a more rapid negative evolution $[7,8]$. A relevant aspect that should be mentioned here is that the metabolic cost of contraction is higher in older than in younger healthy subjects [9]. It is worth noting that most of the previous information has been obtained and refers to limb muscles since specific information on respiratory muscles is almost lacking.

From the tissue and cellular points of view, skeletal muscle ageing mainly entails the reduction of its overall mass due to both atrophy and decrease in the number of fibres (especially type II, which become partly replaced by fat and connective tissue), as well as loss of elasticity, mitochondrial content and capillary fibre ratio, and the disruption of coupling between excitation and contraction (figure 3) [10-12]. 


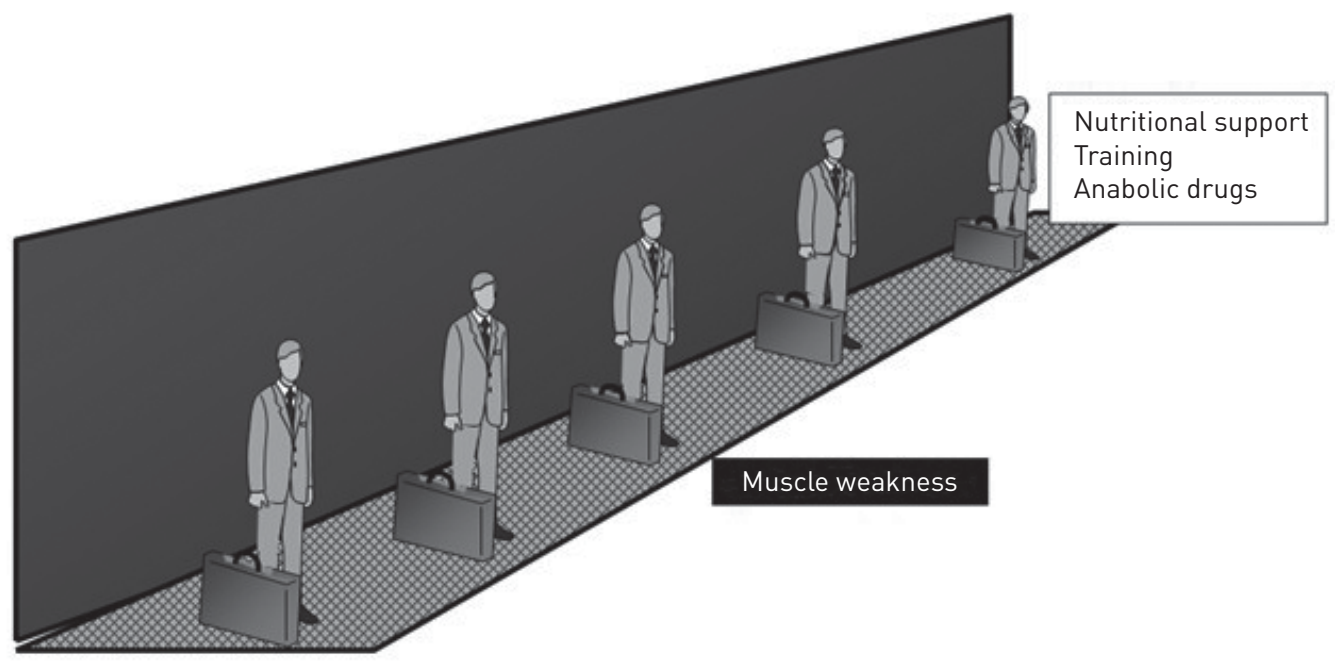

Reserve (against fatigue)

Margin until muscle fatigue (task failure) develops

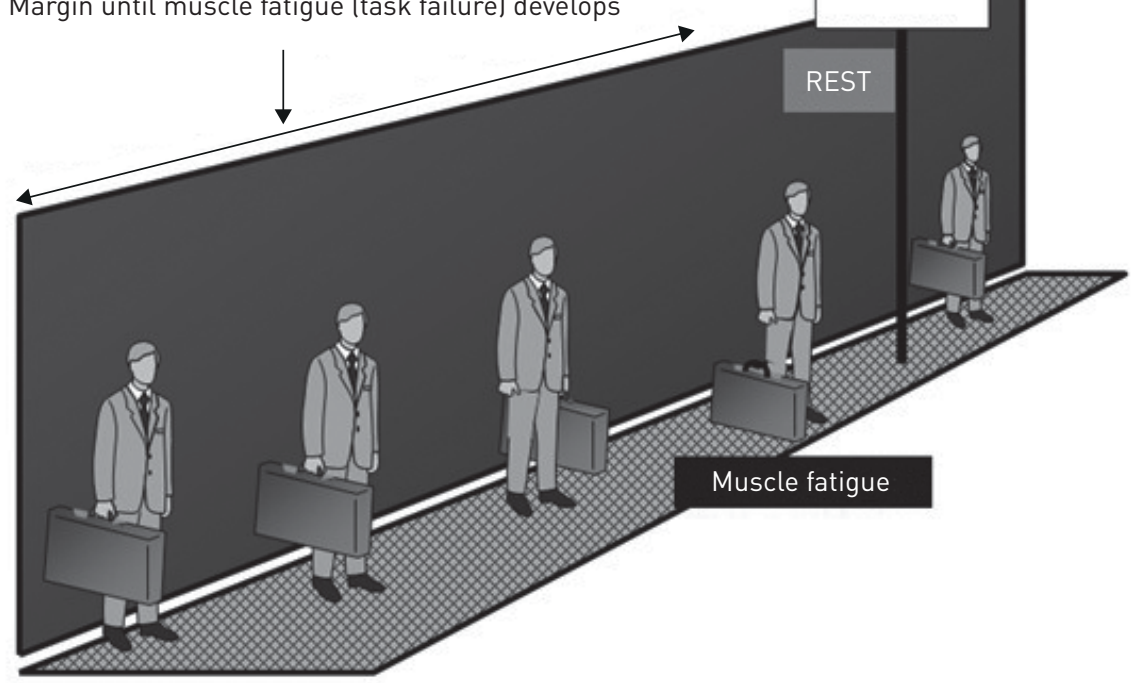

FIGURE 1 Muscle weakness (permanent state) versus muscle fatigue (transient state, solved with rest). NIMV: noninvasive mechanical ventilation; ETIXMV: endotracheal intubation and mechanical ventilation.

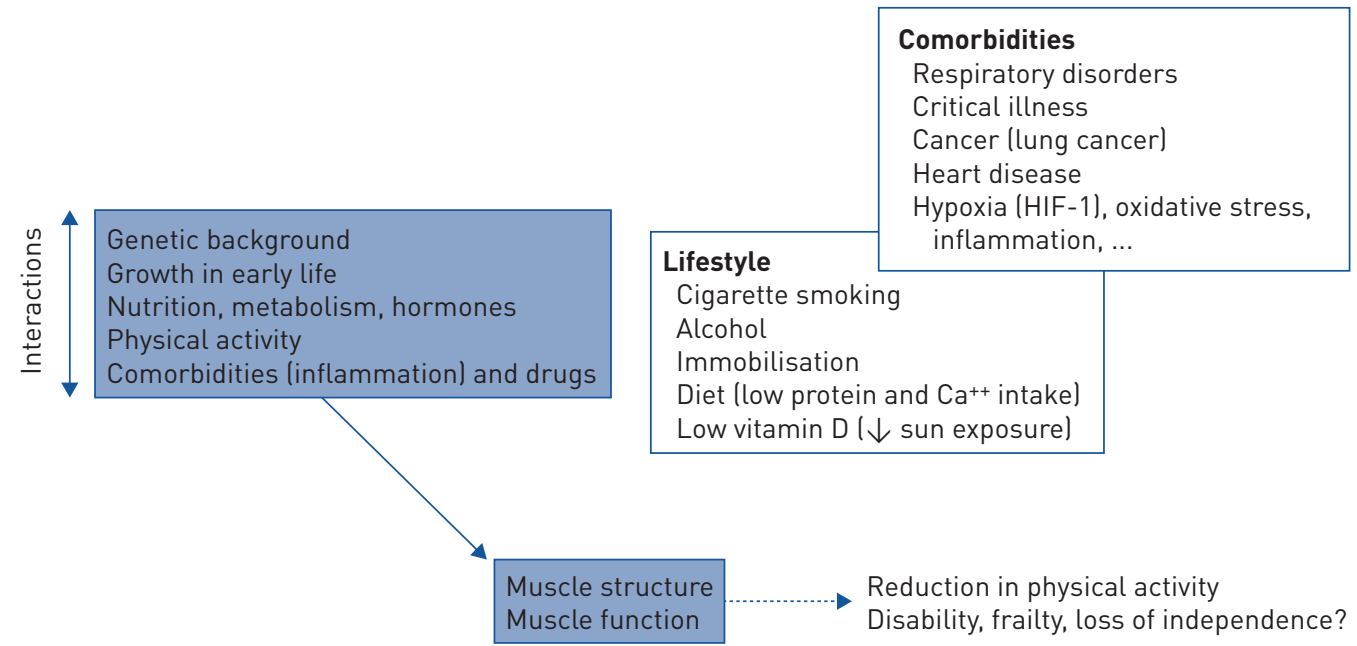

FIGURE 2 Determinants and consequences of muscle ageing. HIF-1: hypoxia-inducible factor-1. 
Healthy ageing

Muscle strength physiologically declines with age

$>50$ years old $\longrightarrow$ Muscle mass $\downarrow$ (1-2\% per year) and muscle strength $\downarrow$ (1.5-3\% per year)

$>65$ years old: sarcopenia $9-18 \%$

$>80$ years old: sarcopenia $30 \%$

Changes in the respiratory system

$\uparrow$ Lung volumes, $\downarrow$ elastic recoil

Stiffening of the chest wall

Broader V' $/ Q$ ' distributions

$\downarrow$ Diffusing capacity

$\downarrow P_{\mathrm{aO}_{2}}, \uparrow P_{\mathrm{A}-\mathrm{aO}}$

Lung immunosenescence

$\downarrow$ Maximim respiratory pressures

\section{Changes in limb muscles}

Oxidative stress, muscle damage

DNA damage, DNA methylation

$\uparrow$ Proteolysis

$\downarrow$ Muscle mass (fat, connective tissue)

$\downarrow$ Type II fibre number and size

Type Ilx fibre denervation

$\downarrow$ Fibre elasticity

$\downarrow$ Capillary/fibre ratio

$\downarrow$ Mitochondrial content and oxidative capacity

个ATP cost for power production

Disruption of excitation/contraction coupling

Stem cell (satellite cell) dysfunction

etc.

\section{Changes in respiratory muscles}

More pronounced oxidative stress

Evidence against (rodents)

Controversial

Mild reduction in muscle mass

Atrophy type II fibres (rodents)

Loss of motoneurons (rodents)

Increased passive stiffness (rodents)

No changes in capillary/fibre (rodents)

Impaired mitochondrial function

Indirect evidence (rodents)

Indirect evidence (rodents)

Evidence against (rodents)

Accelerated ageing

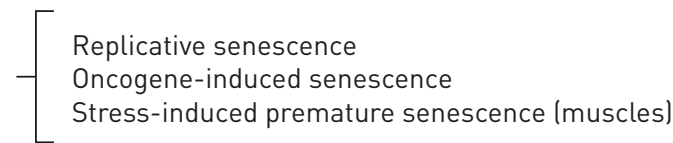

Caused mainly by ROS

Cell changes (flat and larger), dysfunction

Telomere length not affected

FIGURE 3 Healthy versus premature ageing. $V^{\prime} / Q^{\prime}$ : ventilation/perfusion ratio; $P_{\mathrm{aO}_{2}}$ : arterial oxygen tension; $P_{\mathrm{A}-\mathrm{aO}}$ : alveolar-arterial oxygen tension difference; ROS: reactive oxygen species.

All these changes have primarily been studied in human limb muscles or in animal models, since obtaining samples of human respiratory muscles is much more difficult and has numerous ethical and methodological limitations. We know, however, especially from studies in rodents, that the aged diaphragm does not seem to lose excessive muscle mass, but its type IIb and IIx fibres are atrophic, neuromuscular transmission is impaired and its capacity to generate force is therefore reduced [13-15]. The causes of physiological impairment in respiratory muscle function with healthy ageing are multiple and could be classified as either secondary to changes within the respiratory system or those modifications that can be considered as more intrinsic to respiratory muscles, although the latter are in some way often linked to the former. Among the changes occurring with age in the respiratory system, those inherent to the emphysema-like process undergone by the lungs should be highlighted [16]. Therefore, there may be some increase in airway resistances and in static lung volumes, which in turn will lead to greater resistive and elastic work of breathing $[17,18]$. In parallel, ventilation/perfusion $\left(V^{\prime} / Q^{\prime}\right)$ relationships become wider and oxygen diffusion capacity worsens, so gas exchange deteriorates and normal values for arterial oxygen tension are lower than in young individuals (figure 3) $[17,19,20]$. Therefore, the respiratory muscles cope with an increase in loads but under unfavourable geometric dispositions (fundamentally of the diaphragm that would mildly depart from its optimal contraction length) in the presence of a probable lower oxygenation than that of young individuals. Nevertheless, these are not the only deleterious influences present in the elderly; there are other factors that can also be modified by ageing such as the supply of oxygen to the tissues (depending in turn on the haemoglobin content, local blood flow, and vasodilator response to exercise, among others) and its balance with the gas consumption. Moreover, the oxygen flow between capillaries and mitochondria is also very important, and can be affected when there is a decrease in the number of blood vessels or an increase in the distance between them and mitochondria, and/or changes in the number and distribution of the latter, as well as in the amount of myoglobin, all of them potentially occurring with ageing $[12,21,22]$. Furthermore, at the tissue level, there are some studies that demonstrate that older people show higher levels of oxidative and nitrosative stress in their respiratory muscles than in young subjects. A phenomenon that was much more evident in women and not so evident in limb muscles [23]. Another factor that has been involved in the impairment of respiratory muscle function with ageing is the loss of motoneurons in the diaphragm. There is indirect evidence of 
this loss based on the fibre type clustering observed in elderly rodents, and some authors have proposed that this effect could be due to a decrease in the effects of different neurotrophic factors such as the brain-derived neurotrophic factor and neuroregulins, which in normal conditions have anabolic effects on muscle fibres and facilitate neuromuscular transmission [24]. It is interesting to note that, unlike that occurring in the limb muscles, no clear evidence has been found in respiratory muscles of a marked increase in proteolysis, changes in the fibre/capillary ratio, or satellite cell dysfunction.

\section{Comorbidities and ageing}

With regard to respiratory diseases that can normally coexist with ageing, COPD is probably the one where respiratory muscle function and structure have been studied the most. Muscle dysfunction, characterised by the reduction in strength and/or endurance has been repeatedly reported in COPD patients $[1,2,25,26]$. Its causes are multiple and could also be classified as secondary to changes in the respiratory system and those modifications that can be considered as more intrinsic to the muscle. The situation of respiratory muscles in COPD is somewhat similar to that previously mentioned for ageing, but in a much more pronounced way. In other words, changes in the mechanics of the respiratory system and gas exchange impairment will influence it (higher resistances, increased lung volumes with diaphragm shortening, $V^{\prime} / Q^{\prime}$ mismatching, worse oxygen supply to the muscles, etc.), with the possible addition of more specific factors such as tobacco, nutritional abnormalities, exacerbations, marked reduction in physical activity due to respiratory symptoms, systemic inflammation, acidosis and the use of drugs harmful to striated muscles, such as corticosteroids [2]. This accumulation of negative factors can even be enhanced by the concomitant ageing process. Furthermore, as is the case in ageing, it has been consistently described that limb muscles of COPD patients have increased metabolic cost for their activity [27]; an alteration that is not reversible with training. Similar findings have occasionally been observed for respiratory muscles [28].

Regarding the structural and functional findings observed within the respiratory muscles of COPD patients it is known that the diaphragm, intercostal and parasternal muscles show an overall loss of muscle mass [29-31], which seems proportional to respiratory functional deterioration [30, 31]. However, the diaphragm shows a high level of oxidative stress and proteolysis, muscle damage, apoptosis, mitochondrial dysfunction and epigenetic changes [1, 2]. Though, in parallel it also shows apparent beneficial adaptations such as an increase in the proportion of type I fibres (with a predominant aerobic metabolism), capillary/fibre ratio, number of mitochondria, oxidative capacity and myoglobin, as well as shorter sarcomeres $[2,32-34]$. These latter changes probably result from a certain "training effect" due to the increased mechanical loads of the system, and would partially compensate for the aforementioned negative factors $[2,25]$. Similarly, hypoxia that is often associated with respiratory diseases and will be later, also has effects in both directions: on the one hand it can cause oxidative stress and proteolysis, atrophy and fibrillary dysfunction (especially in type II fibres), although on the other hand it also seems to be able to improve mitochondrial efficiency and fatigue tolerance [2, 35-37]. In contrast, in other respiratory conditions, including the critically ill, mechanically ventilated or cancer patients, as well as their corresponding animal models, the diaphragm only shows deleterious effects, with oxidative stress, a high rate of proteolysis, subsequent fibrillar atrophy and functional failure [38-41].

Tissue hypoxia can be present in different respiratory diseases, mainly due to alterations in $V^{\prime} / Q^{\prime}$ relationships and diffusion limitation, but it can also appear in elderly subjects due to other causes such as heart failure or anaemia. The effects of hypoxia on striated muscles are very dependent on the intensity, duration and pattern of the exposure. Upon hypoxia detection by central and arterial chemoreceptors, the expression of inducible factors (hypoxia-inducible factor (HIF), especially HIF-1 $\alpha$ ) increase. These factors control the response of multiple genes, whose task is to adapt tissues to the scarce oxygen available and facilitate the obtaining of energy through the anaerobic pathways. These genes are fundamentally involved in processes such as erythropoiesis, angiogenesis, cell proliferation and survival, inflammation, immune response and autophagy [42]. A variety of molecules, including transcription factors such as nuclear factor- $\mathrm{\kappa B}$, signal transducer and activator of transcription 3, phosphatidylinositol 3 kinase, mitogen-activated protein kinase (MAPK), as well as reactive oxygen species (ROS) and members of the calcium signalling pathways among others, are involved in HIF- $1 \alpha$ regulation $[42,43]$. The production of HIF is also modulated by ageing and associated morbidities and circumstances (e.g. diseases such as respiratory disorders, exercise, altitude, etc.), resulting in a further increase in ROS and subsequent oxidative stress, with changes in DNA and in the mechanisms of cellular and tissue repair, decrease in mitochondrial biogenesis, as well as abnormalities in calcium homeostasis and cell signalling [42, 44]. In fact, HIF- $1 \alpha$ specifically has been involved in premature cellular senescence. Regarding respiratory muscles, in hypoxic conditions they compete for available oxygen with the rest of the body including limb muscles. This can actually lead to different situations depending on the final tissue oxygenation status.If respiratory muscles become hypoxicthe HIF-1 $\alpha$ content will increase, activating MAPK, mTOR, Akt, and 
FoxO3 signalling pathways, which in turn will result in the above mentioned negative effects or in a potential and relatively surprising increase in protein synthesis. Moreover, hypoxia can also increase diaphragm lipid peroxidation [37], redox-sensitive chymotrypsin-like proteasome activity (and proteolysis), and phospho-p38 mitogen-activated protein kinase content [45].

As already mentioned, healthy ageing can produce more mitochondrial dysfunction, ROS production and oxidative stress in respiratory rather than limb muscles $[23,46]$, a difference which can be related to the level of activity/exercise. With respect to the effects of exercise on the respiratory muscles of elderly patients with respiratory diseases, it is known that if it is intense enough it can cause fibrillary injury, associated with oxidative stress and the expression of pro-inflammatory cytokines, which are followed by the expression of markers of regeneration [47]. Moreover, various studies have shown that respiratory muscle training (which is actually a repeated and controlled exercise of moderate-to-high intensity) can improve their structural and functional properties, both in healthy elderly people and COPD patients of similar age [48-50]. It is well known from animal models and human studies that intense exercise can cause respiratory muscle damage, and that if this injury is limited, tissue repair and remodelling will take place $[47,51,52]$, being the substrate of muscle training. In this scenario, it is important to remember that the muscle has its own repair stem cells; the satellite cells [38]. However, ageing leads to a higher ROS production in stem cells in general, with activation of p38 and FoxO, as well as DNA damage and methylation, leading to the impairment of their functions [53]. Nevertheless, some authors have proposed that both the state of inactivity (quiescence) and autophagy are protective mechanisms against the mentioned DNA damage in satellite cells $[54,55]$, although the first of these processes could be lost with ageing [56]. As reviewed here, the same abnormalities observed in stem cells also occur in fibres of the peripheral muscles of older COPD patients [57], and have also been demonstrated in the primary cultures of their own myoblasts [58]. The latter are actually their satellite cells, since muscle fibres, being post-mitotic, do not survive in culture. Another interesting topic is that of exercise and training under hypoxic conditions, a situation that can be quite common in elderly respiratory patients. It is well known that resistance training performed under hypoxia can have beneficial effects in healthy subjects, since it favours the enhancement of the aerobic capacity of the skeletal muscles [59], while it can also increase their strength by increasing protein synthesis and therefore the size of their fibres [60].

\section{Premature senescence}

An interesting phenomenon to be analysed is the so-called accelerated ageing or premature senescence (figure 3). In reality, cells and tissues can undergo three different types of early senescence: 1) replicative; 2) oncogene-induced; and 3) stress-induced premature (SIPS) senescence. The latter is believed to be predominant in striated muscle, and shares some characteristics such as cellular morphology and activity with the replicative senescence, but differs in that the length of telomeres remain unchanged. The main trigger for SIPS is oxidative stress. If this stress is experimentally induced in primary myoblast cultures from young subjects, the expression of up to 40 genes becomes modified, predominantly those genes involved in the metabolism of amines, hormonal biosynthesis and activity of lipases [61]. In a relatively recent study carried out on the peripheral muscles of elderly COPD patients with low weight, an increase in proinflammatory factors, oxidative stress and fibrillar damage, as well as regeneration markers, was observed, along with a typical senescent decrease in DNA repair factors, but with no changes in telomere length [62]. Unfortunately, as yet no results on these same phenomena are available in respiratory muscles of either healthy old individuals or elderly respiratory patients.

In summary, respiratory muscles are striated muscles whose function is to generate changes in the intrathoracic pressure to generate airflow. In the process of senescence respiratory muscles become affected through the general body ageing process and factors that are more specific for these muscles. The former influences the function of respiratory muscles mainly through changes in the respiratory system (increased elastic and resistive work of breathing, worsening of alveolar-capillary diffusion and $V^{\prime} / Q^{\prime}$ ratios, etc.), but also other factors involved in the transport and use of oxygen by the muscles (haematocrit, number of capillaries and distance from them to fibres, local blood flow, etc.). However, there are direct effects of ageing on the respiratory muscles, with changes involving both fibres and satellite cells, among which the presence of oxidative stress, DNA and cellular damage, proteolysis and defects in the repair mechanisms should be highlighted. There is a physiological ageing process in all striated muscles, characterised by the loss of their function. However, the presence of factors such as the presence of concomitant diseases or associated circumstances (such as COPD, lung cancer or hypoxia) can increase the magnitude of these changes. There is also the concept of accelerated senescence, a process that can affect all organs and tissues. In the case of striated muscles, this premature ageing seems to be due primarily to oxidative stress, being considered to be from the SIPS modality. This is characterised by changes in the cellular structure and functions similarly to those observed in replicative senescence, but with no significant effects on 
telomere length. However, there is still a lack of evidence on the existence of SIPS in ventilatory muscles, with or without associated respiratory disorders.

Acknowledgements: The authors thanks Jonathan McFarland (Academic Writing Office, Sechenov University, Moscow, Russia) for his editing assistance.

Conflict of interest: None declared.

\section{References}

1 Laveneziana P, Albuquerque A, Aliverti A, et al. ERS statement on respiratory muscle testing at rest and during exercise. Eur Respir J 2019; 53: 1801214.

2 Gea J, Pascual S, Casadevall C, et al. Muscle dysfunction in chronic obstructive pulmonary disease: update on causes and biological findings. $J$ Thorac Dis 2015; 7: E418-E438.

3 Curtis E, Litwic A, Cooper C, et al. Determinants of muscle and bone aging. J Cell Physiol 2015; 230: 2618-2625.

4 Greising SM, Ottenheijm CAC, O'Halloran KD, et al. Diaphragm plasticity in aging and disease: therapies for muscle weakness go from strength to strength. J Appl Physiol 2018; 125: 243-253.

5 Lang T, Streeper T, Cawthon P, et al. Sarcopenia: etiology, clinical consequences, intervention, and assessment. Osteoporos Int 2010; 21: 543-559.

6 McGregor RA, Cameron-Smith D, Poppitt SD. It is not just muscle mass: a review of muscle quality, composition and metabolism during ageing as determinants of muscle function and mobility in later life. Longev Healthspan 2014; 3: 9.

7 Kortebein P, Ferrando A, Lombeida J, et al. Effect of 10 days of bed rest on skeletal muscle in healthy older adults. JAMA 2007; 297: 1772-1774.

8 Zampieri S, Pietrangelo L, Loefler S, et al. Lifelong physical exercise delays age-associated skeletal muscle decline. J Gerontol A Biol Sci Med Sci 2015; 70: 163-173.

9 Layec G, Trinity JD, Hart CR, et al. Impact of age on exercise-induced ATP supply during supramaximal plantar flexion in humans. Am J Physiol Regul Integr Comp Physiol 2015; 309: R378-R388.

10 Lexell J. Human aging, muscle mass, and fiber type composition. J Gerontol A Biol Sci Med Sci 1995; 50: 11-16.

11 Wang Y, Pessin JE. Mechanisms for fiber-type specificity of skeletal muscle atrophy. Curr Opin Clin Nutr Metab Care 2013; 16: 243-250.

12 Groen BB, Hamer HM, Snijders T, et al. Skeletal muscle capillary density and microvascular function are compromised with aging and type 2 diabetes. J Appl Physiol 2014; 116: 998-1005.

13 Greising SM, Medina-Martínez JS, Vasdev AK, et al. Analysis of muscle fiber clustering in the diaphragm muscle of sarcopenic mice. Muscle Nerve 2015; 52: 76-82.

14 Greising SM, Ermilov LG, Sieck GC, et al. Ageing and neurotrophic signalling effects on diaphragm neuromuscular function. J Physiol 2015; 593: 431-440.

15 Greising SM, Mantilla CB, Gorman BA, et al. Diaphragm muscle sarcopenia in aging mice. Exp Gerontol 2013; 48: 881-887.

16 Skloot GS. The effects of aging on lung structure and function. Clin Geriatr Med 2017; 33: 447-457.

17 Lalley PM. The aging respiratory system--pulmonary structure, function and neural control. Respir Physiol Neurobiol 2013; 187: 199-210.

18 Smith JR, Cross TJ, Van Iterson EH, et al. Resistive and elastic work of breathing in older and younger adults during exercise. J Appl Physiol 2018; 125: 190-197.

19 Cardús J, Burgos F, Diaz O, et al. Increase in pulmonary ventilation-perfusion inequality with age in healthy individuals. Am J Respir Crit Care Med 1997; 156: 648-653.

20 Delclaux B, Orcel B, Housset B, et al. Arterial blood gases in elderly persons with chronic obstructive pulmonary disease (COPD). Eur Respir J 1994; 7: 856-861.

21 Wray DW, Richardson RS. 'Fine-tuning' blood flow to the exercising muscle with advancing age: an update. Exp Physiol 2015; 100: 589-602.

22 Roman MA, Rossiter HB, Casaburi R. Exercise, ageing and the lung. Eur Respir J 2016; 48: 1471-1486.

23 Barreiro E, Coronell C, Laviña B, et al. Aging, sex differences, and oxidative stress in human respiratory and limb muscles. Free Radic Biol Med 2006; 41: 797-809.

24 Elliott JE, Greising SM, Mantilla CB, et al. Functional impact of sarcopenia in respiratory muscles. Respir Physiol Neurobiol 2016; 226: 137-146.

25 Similowski T, Yan S, Gauthier AP, et al. Contractile properties of the human diaphragm during chronic hyperinflation. N Engl J Med 1991; 325: 917-923.

26 Ramírez-Sarmiento A, Orozco-Levi M, Barreiro E, et al. Expiratory muscle endurance in chronic obstructive pulmonary disease. Thorax 2002; 57: 132-136.

27 Sala E, Roca J, Marrades RM, et al. Effects of endurance training on skeletal muscle bioenergetics in chronic obstructive pulmonary disease. Am J Respir Crit Care Med 1999; 159: 1726-1734.

28 Scano G, Grazzini M, Stendardi L, et al. Respiratory muscle energetics during exercise in healthy subjects and patients with COPD. Respir Med 2006; 100: 1896-1906.

29 Arora NS, Rochester DF. COPD and human diaphragm muscle dimensions. Chest 1987; 91: 719-724.

30 Ju S, Lee SJ, Park MJ, et al. Clinical importance of cross-sectional area of intercostal muscles in patients with chronic obstructive pulmonary disease. Clin Respir J 2018; 12: 939-947.

31 Wallbridge P, Parry SM, Das S, et al. Parasternal intercostal muscle ultrasound in chronic obstructive pulmonary disease correlates with spirometric severity. Sci Rep 2018; 8: 15274.

32 Levine S, Kaiser L, Leferovich J, et al. Cellular adaptations in the diaphragm in chronic obstructive pulmonary disease. N Engl J Med 1997; 337: 1799-1806.

33 Orozco-Levi M, Gea J, Lloreta JL, et al. Subcellular adaptation of the human diaphragm in chronic obstructive pulmonary disease. Eur Respir J 1999; 13: 371-378.

34 Wijnhoven JH, Janssen AJ, van Kuppevelt TH, et al. Metabolic capacity of the diaphragm in patients with COPD. Respir Med 2006; 100: 1064-1071. 
35 Gamboa JL, Andrade FH. Mitochondrial content and distribution changes specific to mouse diaphragm after chronic normobaric hypoxia. Am J Physiol Regul Integr Comp Physiol 2010; 298: R575-R583.

36 McMorrow C, Fredsted A, Carberry J, et al. Chronic hypoxia increases rat diaphragm muscle endurance and sodium-potassium ATPase pump content. Eur Respir J 2011; 37: 1474-1481.

37 O'Leary AJ, Drummond SE, Edge D, et al. Diaphragm muscle weakness following acute sustained hypoxic stress in the mouse is prevented by pretreatment with N-acetyl cysteine. Oxid Med Cell Longev 2018; 2018: 4805493.

38 Gea J, Barreiro E, Orozco-Levi M. Skeletal muscle adaptation to disease states. In: R Bottinelli, C Reggiani, eds. Skeletal Muscle Plasticity in Health and Disease: From genes to whole muscle. Doordrecht, Springer, 2006; pp. 315-360.

39 Salazar-Degracia A, Blanco D, Vilà-Ubach M, et al. Phenotypic and metabolic features of mouse diaphragm and gastrocnemius muscles in chronic lung carcinogenesis: influence of underlying emphysema. J Transl Med 2016; 14: 244.

40 Fermoselle C, García-Arumí E, Puig-Vilanova E, et al. Mitochondrial dysfunction and therapeutic approaches in respiratory and limb muscles of cancer cachectic mice. Exp Physiol 2013; 98: 1349-1365.

41 Marin-Corral J, Dot I, Boguña M, et al. Structural differences in the diaphragm of patients following controlled vs assisted and spontaneous mechanical ventilation. Intensive Care Med 2019; 45: 488-500.

42 Yeo EJ. Hypoxia and aging. Exp Mol Med 2019; 51: 1-15.

43 Koh MY, Spivak-Kroizman TR, Powis G. HIF-1 regulation: not so easy come, easy go. Trends Biochem Sci 2008; 33: 526-534.

44 Yuan Y, Cruzat VF, Newsholme P, et al. Regulation of SIRT1 in aging: roles in mitochondrial function and biogenesis. Mech Ageing Dev 2016; 155: 10-21.

45 Lewis P, Sheehan D, Soares R, et al. Redox remodeling is pivotal in murine diaphragm muscle adaptation to chronic sustained hypoxia. Am J Respir Cell Mol Biol 2016; 55: 12-23.

46 Puente-Maestu L, Pérez-Parra J, Godoy R, et al. Abnormal mitochondrial function in locomotor and respiratory muscles of COPD patients. Eur Respir J 2009; 33: 1045-1052.

47 Casadevall C, Coronell C, Ramirez-Sarmiento AL, et al. Effects of a bout of exhaustive ventilatory effort in the intercostal muscles of severe COPD: expression profiles of local inflammatory and regeneration markers. Stockholm (S). Eur Respir J 2007; 30: Suppl. 51, 727s.

48 Ramirez-Sarmiento A, Orozco-Levi M, Guell R, et al. Inspiratory muscle training in patients with chronic obstructive pulmonary disease: structural adaptation and physiologic outcomes. Am J Respir Crit Care Med 2002; 166: 1491-1497.

49 Souza $\mathrm{H}$, Rocha $\mathrm{T}$, Pessoa $\mathrm{M}$, et al. Effects of inspiratory muscle training in elderly women on respiratory muscle strength, diaphragm thickness and mobility. J Gerontol A Biol Sci Med Sci 2014; 69: 1545-1553.

50 Alvarenga GM, Charkovski SA, Santos LKD, et al. The influence of inspiratory muscle training combined with the Pilates method on lung function in elderly women: a randomized controlled trial. Clinics (Sao Paulo) 2018; 73: e356.

51 Zhu E, Petrof BJ, Gea J, et al. Diaphragm muscle fiber injury after inspiratory resistive breathing. Am J Respir Crit Care Med 1997; 155: 1110-1116.

52 Gea J, Hamid Q, Czaika G, et al. Expression of myosin heavy-chain isoforms in the respiratory muscles following inspiratory resistive breathing. Am J Respir Crit Care Med 2000; 161: 1274-1278.

53 Yang TT, Lo CP, Tsai PS, et al. Aging and exercise affect hippocampal neurogenesis via different mechanisms. PLoS One 2015; 10: e0132152.

54 García-Prat L, Martínez-Vicente M, Perdiguero E, et al. Autophagy maintains stemness by preventing senescence. Nature 2016; 529: 37-42.

55 Valenti MT, Dalle Carbonare L, Dorelli G, et al. Effects of physical exercise on the prevention of stem cells senescence. Stem Cell Rev Rep 2020; 16: 33-40.

56 Sousa-Victor P, Gutarra S, García-Prat L, et al. Geriatric muscle stem cells switch reversible quiescence into senescence. Nature 2014; 506: 316-321.

57 Puig-Vilanova E, Rodriguez DA, Lloreta J, et al. Oxidative stress, redox signaling pathways, and autophagy in cachectic muscles of male patients with advanced COPD and lung cancer. Free Radic Biol Med 2015; 79: 91-108.

58 Gouzi $\mathrm{F}$, Blaquière $\mathrm{M}$, Catteau $\mathrm{M}$, et al. Oxidative stress regulates autophagy in cultured muscle cells of patients with chronic obstructive pulmonary disease. J Cell Physiol 2018; 233: 9629-9639.

59 Keramidas ME, Debevec T, Amon M, et al. Respiratory muscle endurance training: effect on normoxic and hypoxic exercise performance. Eur J Appl Physiol 2010; 108: 759-769.

60 Fernández-Lázaro D, Díaz J, Caballero A, et al. The training of strength-resistance in hypoxia: effect on muscle hypertrophy. Biomedica 2019; 39: 212-220.

61 Lim JJ, Wan Zurinah WN, Mouly V, et al. Tocotrienol-rich fraction (TRF) treatment promotes proliferation capacity of stress-induced premature senescence myoblasts and modulates the renewal of satellite cells: microarray analysis. Oxid Med Cell Longev 2019; 2019: 9141343.

62 Lakhdar R, McGuinness D, Drost EM, et al. Role of accelerated aging in limb muscle wasting of patients with COPD. Int J Chron Obstruct Pulmon Dis 2018; 13: 1987-1998. 